\title{
Influence of Age and Sex on Disease Course and Treatment in Rheumatoid Arthritis
}

\author{
Jenny Nilsson' \\ Maria LE Andersson (1D ${ }^{2,3}$ \\ Ingiäld Hafström ${ }^{4}$ \\ Björn Svensson $\mathbb{D D}^{2}$ \\ Kristina Forslind $\mathbb{D D}^{2,3}$ \\ Sofia Ajeganova ${ }^{4,5}$ \\ Monica Leu Agelii (D) ${ }^{1, *}$ \\ Inger Gjertsson ${ }^{1} *$
}

'Department of Rheumatology and Inflammation Research, Institute of Medicine, Sahlgrenska Academy, Gothenburg University, Gothenburg, Sweden; ${ }^{2}$ Lund University, Faculty of Medicine, Department of Clinical Sciences Lund, Rheumatology, Lund, Sweden; ${ }^{3}$ Spenshult Research and

Development Center, Halmstad, Sweden;

${ }^{4}$ Division of Gastroenterology and Rheumatology, Department of Medicine Huddinge, Karolinska Institutet, and Karolinska University Hospital, Stockholm, Sweden; ${ }^{5}$ Department of Clinical Sciences, Rheumatology Division. Universitair Ziekenhuis Brussel, Vrije Universiteit Brussel, Brussels, Belgium

*These authors contributed equally to this work

Correspondence: Monica Leu Agelii Department of Rheumatology and Inflammation Research, Institute of Medicine, Sahlgrenska Academy, Gothenburg University, Box 480, Gothenburg, 40530, Sweden

Tel +46- 3I-3424692

Email monica.leu@gu.se
Objective: More than $50 \%$ of patients with rheumatoid arthritis (RA) are $>65$ years at diagnosis. Age of onset and sex may influence the disease course, outcome and treatment. This study follows a large cohort of patients with early RA to assess contributions of age and sex to disease outcomes.

Methods: Patients from the BARFOT cohort, $n=2837$ (68\% women), were followed for eight years at predefined time points to assess inflammation, function, joint destruction and treatment with disease modifying anti-rheumatic drugs (DMARDs) and glucocorticoids (GC). The patients were divided by sex and age at inclusion $(<40,40-54,55-69$ and $\geq 70$ years).

Results: For both sexes, disease activity, function and pain improved over time, significantly more in men than in women in all age groups. In men, those $<40$ years displayed significantly lower DAS28 compared with all other groups. This group was also the least represented group in the study. The Sharp van der Heijde Score (SHS) increased over time in both sexes and all age groups. Women $\geq 70$ years showed less improvement in disability and the highest progression of SHS mainly due to increased joint space narrowing. Patients $<40$ years were more likely to receive biological DMARDs, while those $\geq 70$ years more often received only GC treatment.

Conclusion: There were significant age- and sex-dependent differences in the medical treatment and in outcome of RA 8 years after diagnosis. The differences were most pronounced in men $<40$ and women $\geq 70$ years, but whether they are due to disease phenotype or treatment is unclear.

Keywords: rheumatoid arthritis, disease course, treatment, age- and sex-dependent differences

\section{Plain Language Summary}

Rheumatoid arthritis (RA) is a chronic inflammatory joint disease that often results in joint destruction and disability. It affects approximately $1 \%$ of the population and more often women than men. The demographics of the Western world are changing and today, more than $50 \%$ of RA patients are older than 65 years at diagnosis.

The aim of this study was to look at the influence of age and sex on the outcome and treatment of RA. To this end, we have examined a cohort of 2837 RA patients from disease onset and over the following 8 years.

The patients were divided into groups by sex and age at inclusion $(<40,40-54,55-69$ and $\geq 70$ years). For all patients, disease activity and pain improved over time, but joint destruction increased. Generally, men were doing better compared to women. Women $\geq 70$ years had a more destructive disease compared with all other patients. Biologic antirheumatic drugs were introduced during the collection of the cohort and were mainly 
prescribed to younger patients, preferentially men. Less than $20 \%$ of the patients were treated with glucocorticoids only, but the majority of these patients were $\geq 70$ years.

We suggest that advanced age, especially for women, should be considered a risk factor for worse disease outcome and that specific treatment guidelines are desirable for patients $\geq 70$ years at disease onset.

\section{Introduction}

Rheumatoid arthritis (RA) is a chronic inflammatory joint disease that often results in joint destruction and disability. The prevalence is $0.5-1 \%$ in European and NorthAmerican populations. In Sweden, the prevalence of RA is $0.77 \%$ and the overall incidence is 41 per 100,000 inhabitants, which peaks between $70-79$ years. The prevalence of RA is 2-3 times higher in women than in men, but this ratio decreases with age at onset. ${ }^{1}$ Women generally have a worse outcome than men with respect to disease activity ${ }^{2,3}$ and disability, ${ }^{3}$ while joint destruction is the same for women and men. ${ }^{2,4}$ The demography in the Western world is changing and more than $50 \%$ of all patients with RA are over 65 years of age at diagnosis. ${ }^{1}$ Aging is associated with increased prevalence of cardiovascular disease and osteoporosis, both of which are overrepresented as comorbidities in patients with RA compared with the general population. ${ }^{4}$ They contribute, alongside RA per se, to the impairment of quality of life, and this combination of morbidities magnifies the socioeconomic costs of supporting patients with RA relative to healthy individuals. ${ }^{5}$

International treatment guidelines recommend that treatment with disease modifying anti-rheumatic drugs (DMARDs) at diagnosis, irrespective of sex and age and the presence of poor prognostic factors, should be pursued actively. ${ }^{6,7}$ Although the guidelines do not discriminate on the basis of sex or age, younger patients seem to receive more treatment with both conventional synthetic (cs) and biological (b) DMARDs, ${ }^{8-10}$ whereas elderly patients are more likely to receive glucocorticoids (GC) only ${ }^{11}$ and less likely to receive DMARD ${ }^{12}$ especially bDMARDs. ${ }^{11-14}$

Previous studies found that age at diagnosis and sex may influence the choice of treatment in RA. However, they were performed during different time periods, had varying lengths of follow-up and varying age stratifications, suggesting a need for further studies. Long-term follow-up studies are necessary to study the disease outcome. However a long-term study means that changes in treatment strategies as well as classification criteria may occur. In this study, the patients were grouped according to sex and age at inclusion and followed for eight years. The rationale for the age group division at disease onset was based on estimated sex hormone levels. Women have the highest incidence of RA around the age of menopause and for men there is an increase in incidence after 40 years of age. $^{2}$ Sex hormones are believed to contribute to the pathogenesis of RA. The age groups were: $<40$ years (no menopause; normal testosterone levels), 40-54 years (woman enters menopause; testosterone levels might start to slowly decrease), 55-69 years (all women have entered menopause; testosterone levels continue to slowly decrease) and $\geq 70$ years (other factors such as comorbidities become important). ${ }^{2}$ In this paper we examined the impact of age and sex on the disease course as to disease activity, function, joint destruction and treatment in the BARFOT (Better Anti Rheumatic Pharmaco Therapy) long-term cohort that included patients with early RA.

\section{Method}

\section{Patients}

This study included 2837 BARFOT patients (1916 women and 921 men). BARFOT is a multicenter longitudinal observational study of RA patients in southern Sweden. ${ }^{15}$ In short, patients with newly diagnosed RA were consecutively included between 1992 and 2006; inclusion criteria were disease duration of 12 months or less and fulfilling the ACR 1987 classification criteria. ${ }^{16}$ The patients were assessed at inclusion, 3 and 6 months, at 1,2,5 and 8 years according to the same structured protocol, see "Clinical assessments" below. Assessments of the 15-year outcomes are ongoing. Smoking habits (current, previous or never smoker) and rheumatic factor (RF) according to respective hospital standards were assessed at inclusion. As antibodies to cyclic citrullinated peptides (anti-CCP) were not routinely collected at the standard timepoints, for the majority of patients they were determined subsequently from serum samples taken at inclusion. Seropositive patients were defined as being RF positive, anti-CCP positive or positive for both these markers, whereas seronegative patients were those negative for both, or RF negative with anti-CCP status unknown.

The patients were grouped according to sex and age at inclusion. The age groups were: $<40$ years, $40-54$ years, $55-69$ years and $\geq 70$ years. 


\section{Clinical Assessments}

Disease activity was assessed through the composite disease activity score (DAS28), calculated on swollen and tender 28-joint counts (SJC and TJC), erythrocyte sedimentation rate (ESR) and patient's assessment of global health on a visual analogue scale (GH-VAS) range 0-100 mm (best to worst). ${ }^{17}$ Similarly, patients reported pain on a VAS (pain-VAS) and functional disability using the Swedish version of Stanford Health Assessment Questionnaire, HAQ, range $0-3{ }^{18}$ The radiographs of hands and feet were scored chronologically using the Sharp van der Heijde scoring method (SHS) by two experienced readers who were blinded to clinical data. The SHS provides separate scores for joint space narrowing (JSN) and erosions (erosion score, ES). ${ }^{19}$

\section{Medical Treatment}

Treatments were registered as: no DMARD, methotrexate only, sulfasalazine only, other csDMARD, combination of at least two csDMARDs or bDMARD alone or in combination. Whether or not the patient received GC was also recorded. At inclusion, the patients were treated with DMARDs in accordance with the recommended therapy strategy in Sweden at that time. This strategy specified in early 1990s initial DMARD monotherapy and early use of low-dose GC, and a "step-up" combination therapy reserved for those with a more severe disease, and from early 2000 s recommended the possibility to initiate treatment with biologics when the first DMARD or combination of DMARDs failed.

\section{Ethics Approval and Consent to Participate}

The study complied with the Declaration of Helsinki and was approved by the Regional Ethical Review Boards at Gothenburg University (Ggb Ö 282-01), Lund University (398-01) and Karolinska Institute (02-075, T2016/297-31/1). Informed, written consent was obtained from the participants before enrolment.

\section{Statistical Analysis}

The patients were grouped according to sex and age at inclusion ( $<40$ years, $40-54,55-69$ and $\geq 70$ years). For each sex, the median for continuous variables was compared between age groups with the Kruskal-Wallis test at inclusion, 1 and 8 years. At the same time points, categorical variables were compared according to the chi-square of independence. To facilitate comparisons with other published studies, the mean values are also presented. For continuous variables, $95 \%$ confidence interval of the median was calculated using bootstrap samples (2000 samples generated with replacement). For proportions, the standard error of proportion was used. To counteract inflation of type I error due to multiple testing, a Bonferroni-Holm ${ }^{20}$ correction was used for each time point individually. The $\mathrm{p}$-values are ranked from smallest to highest and each p-value is compared against a significance level that is adjusted for the number of multiple tests as well as the rank of the p-value: $\alpha$ (rank) $=\alpha /(n-r a n k+1)$, where $\alpha$ is set to 0.05 and $n$ is the number of multiple tests. The analyses included all patients, regardless of whether they had been assessed at all timepoints or not. The term significant is used for differences that are statistically significant at a $95 \%$ confidence level.

All statistical analyses were conducted with MATLAB R2015a (MATLAB and Statistics Toolbox Release 2015a, The MathWorks, Inc., Natick, Massachusetts, United States) and SPSS Statistics Version 25 (IBM Corporation).

\section{Results \\ General Characteristics of the Study Population at Onset of Disease}

Figure 1 gives an overview of the number of participants with available DAS28 data at each follow-up time together with causes of exclusion between the visits. Of the participants present at inclusion, $22 \%$ were not available for the 8 -year examination. Among the 624 patients who were not assessed at 8 years, the main causes for non-participation were death $(70 \%)$, unwillingness to participate $(9 \%)$, development of other disease $(6 \%)$, relocation $(6 \%)$, and unknown causes $(9 \%)$. The non-participating patients were on average 11 years older at inclusion (mean age 66 years, $\mathrm{p}<0.001)$, more likely to be men ( $27 \%$ of the men vs $20 \%$ of the women were missing, $\mathrm{p}<0.001)$. An additional 265 patients had missing DAS28 data at this time point even though they were still included in the study (no information available regarding the cause of missing data).

The patients in this new onset RA cohort had a median age of 60 years (range 15-93 years) at inclusion, $68 \%$ were women and 67\% were seropositive (Table 1). In total, 33\% were current smokers. The median DAS28 score of participants at inclusion was 5.3, containing a median presentation of 7 tender joints and 10 swollen joints. The most represented age group among both 


\section{Inclusion}

2837 patients (1916 women, 921 men) consecutively included between 1992 and 2006 at multiple centers in southern Sweden.

\section{Inclusion criteria:}

Disease duration: less than 12 months

Fulfill the ACR 1987 classification criteria for RA

Exclusion criteria: not fulfilling the inclusion criteria

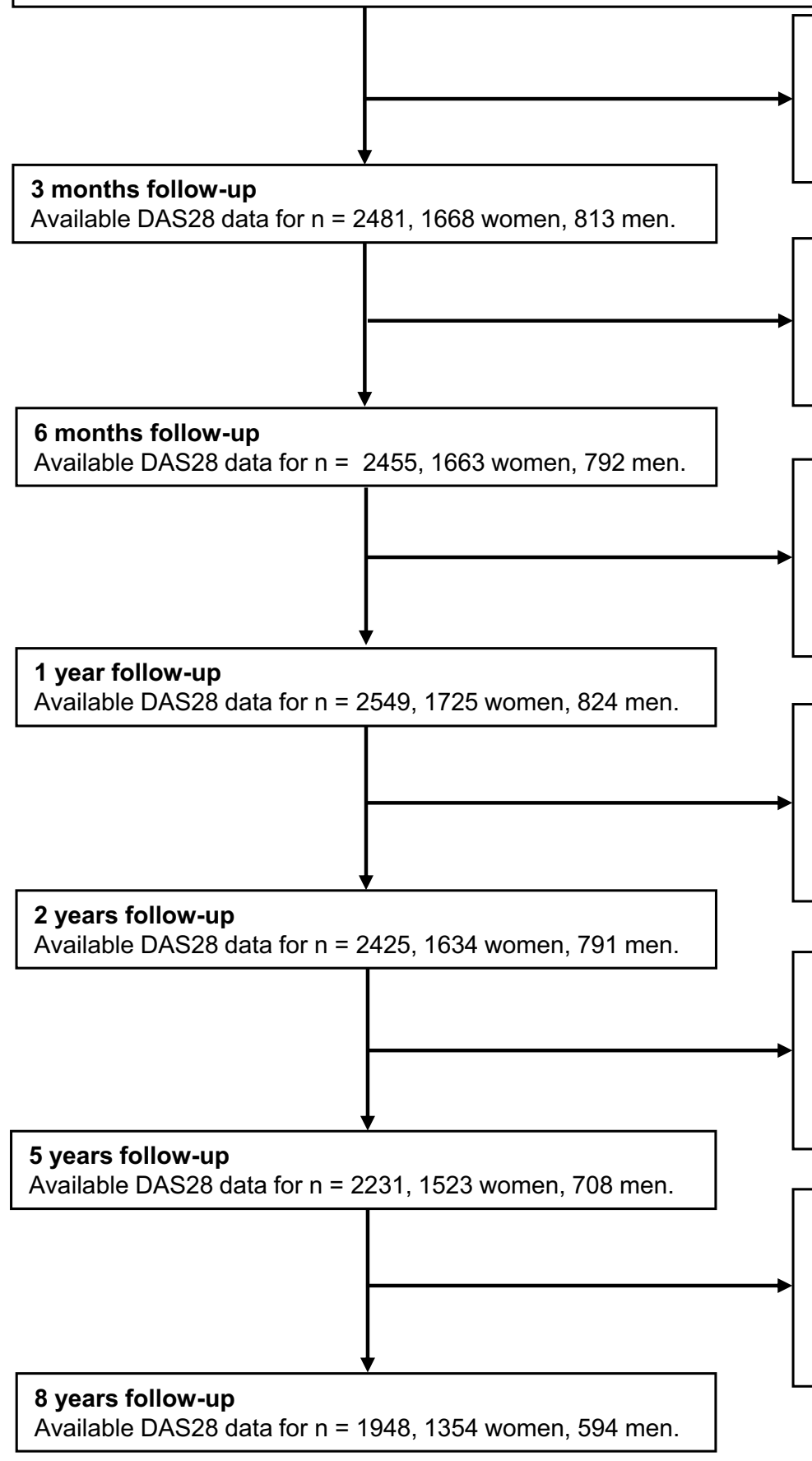

Excluded $n=28$ due to:

Other diseases 4

Death 13

Refusal to participate 4

Other causes 7

Available DAS28 data for $n=2481,1668$ women, 813 men

Excluded $\mathrm{n}=59$ due to:

Other diseases 8

Death 37

Relocation 2

Refusal to participate 6

Other causes 6

Excluded $n=79$ due to:

Other diseases 8

Death 49

Relocation 5

Refusal to participate 6

Other causes 11

Excluded $\mathrm{n}=206$ due to:

Other diseases 8

Death 140

Relocation 20

Refusal to participate 19

Other causes 19

Excluded $n=233$ due to:

Other diseases 7

Death 185

Relocation 13

Refusal to participate 19

Other causes 9

Figure I Overview of the number of patients with available DAS28 data at each time point and causes of exclusion. The number of excluded patients between each time point is registered, and excluded patients did not enter the study again. Some patients with missing information at a certain time point have available data at later visits and are included at those visits. No data are available for the cause of absence at a follow-up. 
Table I Demographic, Clinical and Radiographic Characteristics of All Patients, and Divided into Women and Men at Inclusion

\begin{tabular}{|c|c|c|c|}
\hline & All & Women & Men \\
\hline $\mathrm{N}$ & 2837 & 1916 & 921 \\
\hline Age, years & $60[59,60]$ & $58[57,59]$ & $62[61,64]$ \\
\hline Disease duration, month & $6[6,6]$ & $6[6,6]$ & $6[5,6]$ \\
\hline Seropositive, n (\%) & $67 \%$ & $67 \%$ & $66 \%$ \\
\hline Smoking status & $(n=2698)$ & $(n=1818)$ & $(n=880)$ \\
\hline Current smokers, $\%$ & $33 \%$ & $28 \%$ & $43 \%$ \\
\hline Previous smokers, $\%$ & $27 \%$ & $26 \%$ & $27 \%$ \\
\hline Never smokers, \% & $40 \%$ & $46 \%$ & $30 \%$ \\
\hline DAS28 (0-9.6) & $5.3[5.2,5.4]$ & $5.3[5.3,5.4]$ & $5.2[5.1,5.3]$ \\
\hline Tender joints (0-28) & $7[7,7]$ & $7[7,8]$ & $6[6,7]$ \\
\hline Swollen joints (0-28) & $10[9,10]$ & $10[9,10]$ & $10[10,11]$ \\
\hline $\mathrm{ESR}, \mathrm{mm} / \mathrm{h}$ & $30[28,31]$ & $30[28,31]$ & $29[27,32]$ \\
\hline GH-VAS (0-100) & $46[45,47]$ & $48[47,49]$ & $42[40,45]$ \\
\hline Pain-VAS (0-100) & $47[46,48]$ & $48[47,50]$ & $42[40,45]$ \\
\hline HAQ (0-3) & $1.0[0.9,1.0]$ & $1.0[1.0,1.0]$ & $0.9[0.8 .0 .9]$ \\
\hline SHS (0-448) & I $[0.5,2]$ & I $[0,1]$ & $2[1,2]$ \\
\hline Erosion score (0-280) & $0[0,0]$ & $0[0,0]$ & $0[0,0]$ \\
\hline Joint space narrowing $(0-168)$ & $0[0,0]$ & $0[0,0]$ & $0[0,0]$ \\
\hline
\end{tabular}

Notes: Data are reported as median [95\% lower, upper confidence limits] for continuous variables and as percentages for seropositivity and smoking status. Abbreviations: DAS28, 28-joint disease activity score; ESR, erythrocyte sedimentation rate; GH-VAS, global health on a visual analogue scale; VAS, visual analogue scale; HAQ, Health Assessment Questionnaire; SHS, Sharp van der Heijde Score for joint destruction.

women and men was the 55-69 years-old, with 634 (33\%) women and 355 (39\%) men. The least represented group were younger men, $n=78$ ( $9 \%$ of the male participants). The ratio of women to men decreased with age, being $4: 1$ in the youngest age group falling to less than 2:1 among those older than 70 years (Tables 2 and 3). Sex differences are summarized in Table 4.

\section{Autoantibodies, Symptom Duration Before Diagnosis and Smoking Habits}

The results are shown separately in women (Table 2) and men (Table 3). The majority of the patients studied were seropositive, with the lowest proportions in the age group $\geq 70$ years (women 56\%: men 51\%) and the highest in the age group 40-54 years, (women 73\%: men 78\%). The median time between self-reported onset of symptoms and inclusion was shortest for women and men $\geq 70$ years, and longest for women $<40$ years. Smoking habits at inclusion differed significantly between age groups for both women and men: for both sexes, the proportion of "never smokers" was largest in the age group $<40$ years (57\%). Except for those $<40$ years, more men than women were smokers. The proportion of "current smokers" within age groups differed between the sexes, with most women smokers in the age group 55-69 years (32\%) and most men smokers in the $\geq 70$ age group $(50 \%)$.

\section{Disease Activity Measured as DAS28 and Its Components}

Figure 2 shows the median DAS28 for women (A) and men (B) over 8 years from diagnosis. The number of participants on which the DAS28 statistics were calculated are presented in Supplementary Table 1, by sex and age group. In all age groups and for both sexes DAS28, as well as number of tender and swollen joints, decreased most during the first year after inclusion in the study. At inclusion, DAS28 differed significantly between age groups for women $((\mathrm{p}<0.001$, Figure $2 \mathrm{~A}$ and Table 4$)$ and women aged older than 55 years had the highest DAS28 (median 5.5, Table 4). For men, DAS28 at inclusion did not vary 
Table 2 Clinical Measures for Women at Inclusion, I Year and 8 Years

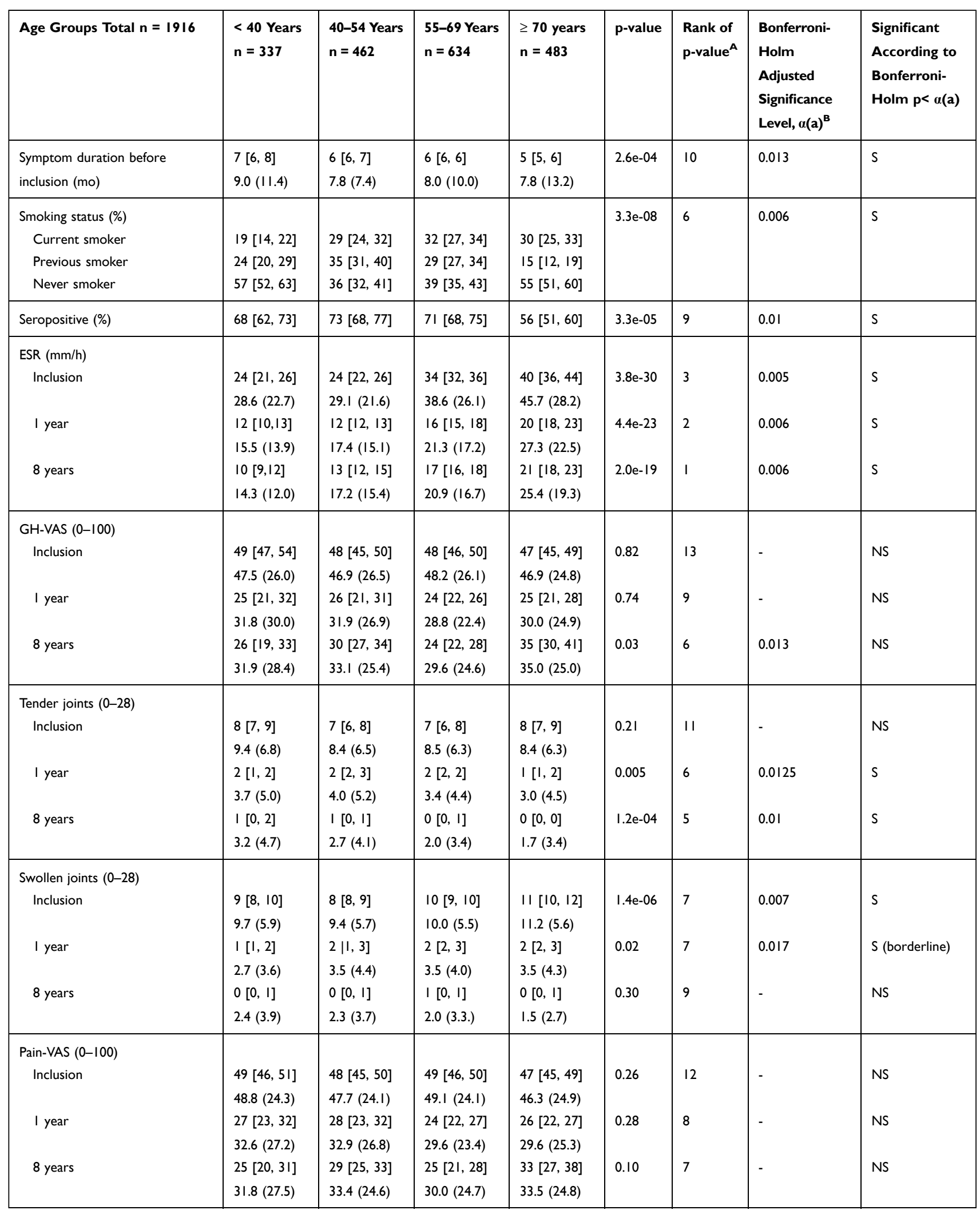

(Continued) 
Table 2 (Continued).

\begin{tabular}{|c|c|c|c|c|c|c|c|c|}
\hline Age Groups Total $n=1916$ & $\begin{array}{l}<40 \text { Years } \\
n=337\end{array}$ & $\begin{array}{l}40-54 \text { Years } \\
n=462\end{array}$ & $\begin{array}{l}55-69 \text { Years } \\
n=634\end{array}$ & $\begin{array}{l}\geq 70 \text { years } \\
n=483\end{array}$ & p-value & $\begin{array}{l}\text { Rank of } \\
\text { p-value }\end{array}$ & $\begin{array}{l}\text { Bonferroni- } \\
\text { Holm } \\
\text { Adjusted } \\
\text { Significance } \\
\text { Level, } \alpha(a)^{B}\end{array}$ & $\begin{array}{l}\text { Significant } \\
\text { According to } \\
\text { Bonferroni- } \\
\text { Holm p< } \alpha(a)\end{array}$ \\
\hline \multicolumn{9}{|l|}{ HAQ (0-3) } \\
\hline Inclusion & $\begin{array}{l}0.9[0.8,1.0] \\
1.0(0.6)\end{array}$ & $\begin{array}{l}1.0[0.9,1.0] \\
1.0(0.6)\end{array}$ & $\begin{array}{l}1.0[1.0,1.0] \\
1.1(0.6)\end{array}$ & $\begin{array}{l}\text { I.3[1.I, I.4] } \\
1.3(0.7)\end{array}$ & $3.4 \mathrm{e}-09$ & 5 & 0.006 & S \\
\hline I year & $\begin{array}{l}0.4[0.3,0.5] \\
0.6(0.6)\end{array}$ & $\begin{array}{l}0.6[0.5,0.8] \\
0.7(0.6)\end{array}$ & $\begin{array}{l}0.6[0.5,0.7] \\
0.7(0.6)\end{array}$ & $\begin{array}{l}0.8[0.6,0.9] \\
0.8(0.7)\end{array}$ & $1.2 \mathrm{e}-05$ & 4 & 0.008 & S \\
\hline 8 years & $\begin{array}{l}0.4[0.3,0.5] \\
0.6(0.6)\end{array}$ & $\begin{array}{l}0.6[0.5,0.8] \\
0.7(0.6)\end{array}$ & $\begin{array}{l}0.6[0.6,0.7] \\
0.7(0.6)\end{array}$ & $\begin{array}{l}0.9[0.6,1.0] \\
1.0(0.8)\end{array}$ & $1.5 \mathrm{e}-10$ & 3 & 0.007 & $S$ \\
\hline \multicolumn{9}{|l|}{ Joint destruction (SHS) } \\
\hline \multicolumn{9}{|l|}{ Total score $(0-448)$} \\
\hline Inclusion & $\begin{array}{l}0[0,0] \\
1.6(3.9)\end{array}$ & $\begin{array}{l}0[0,0] \\
3.4(7.6)\end{array}$ & $\begin{array}{l}2[1,3] \\
5.9(9.4)\end{array}$ & $\begin{array}{l}4[3,5] \\
8.5(11.6)\end{array}$ & $2.9 e-33$ & 2 & 0.004 & S \\
\hline I year & $\begin{array}{l}0[0,1] \\
4.5(7.7)\end{array}$ & $\begin{array}{l}2[1,3] \\
7.1(11.8)\end{array}$ & $\begin{array}{l}5[4,6] \\
10.0(12.9)\end{array}$ & $\begin{array}{l}8[7,11] \\
13.3(15.6)\end{array}$ & I.le-22 & 3 & 0.007 & S \\
\hline 8 years & $\begin{array}{l}5[3,7] \\
14.6(21.8)\end{array}$ & $\begin{array}{l}\text { II }[9,14] \\
20.2(27.0)\end{array}$ & $\begin{array}{l}15[12,18] \\
24.3(26.5)\end{array}$ & $\begin{array}{l}19[14,21] \\
26.6(27.3)\end{array}$ & $9.8 \mathrm{e}-10$ & 4 & 0.008 & S \\
\hline \multicolumn{9}{|l|}{ Erosion score $(0-280)$} \\
\hline Inclusion & $\begin{array}{l}0[0,0] \\
0.7(1.8)\end{array}$ & $\begin{array}{l}0[0,0] \\
\text { I.2(3.5) }\end{array}$ & $\begin{array}{l}0[0,0] \\
\text { I.6 (3.2) }\end{array}$ & $\begin{array}{l}0[0,0] \\
1.9(3.9)\end{array}$ & $3.5 \mathrm{e}-06$ & 8 & & S \\
\hline I year & $\begin{array}{l}0[0,0] \\
1.9(3.5)\end{array}$ & $\begin{array}{l}0[0,0.5] \\
2.5(4.9)\end{array}$ & $\begin{array}{l}1[0.5,1] \\
3.0(4.9)\end{array}$ & $\begin{array}{l}0.5[0,1] \\
3.4(5.9)\end{array}$ & 0.001 & 5 & 0.01 & s \\
\hline 8 years & $\begin{array}{l}\text { I }[0,2] \\
5.3(8.6)\end{array}$ & $\begin{array}{l}2[1,3] \\
6.6(11.9)\end{array}$ & $\begin{array}{l}2[1,3] \\
6.8(9.9)\end{array}$ & $\begin{array}{l}\text { I }[1,2] \\
6.7(9.9)\end{array}$ & 0.12 & 8 & - & NS \\
\hline \multicolumn{9}{|l|}{ Joint space narrowing (0-168) } \\
\hline Inclusion & $\begin{array}{l}0[0,0] \\
0.9(2.7)\end{array}$ & $\begin{array}{l}0[0,0] \\
2.2(5.3)\end{array}$ & $\begin{array}{l}0[0,1] \\
4.3(7.3)\end{array}$ & $\begin{array}{l}4[3,5] \\
6.6(8.9)\end{array}$ & $5.0 e-40$ & 1 & 0.004 & $\mathrm{~S}$ \\
\hline I year & $\begin{array}{l}0[0,0] \\
2.6(5.4)\end{array}$ & $\begin{array}{l}0[0,1] \\
4.7(8.6)\end{array}$ & $\begin{array}{l}3[2,4] \\
7.0(9.8)\end{array}$ & $\begin{array}{l}6[4,7] \\
9.9(11.5)\end{array}$ & $4.2 \mathrm{e}-29$ & 1 & 0.006 & $S$ \\
\hline 8 years & $\begin{array}{l}3[0,4] \\
9.4(14.3)\end{array}$ & $\begin{array}{l}8[6,10] \\
13.6(17.2)\end{array}$ & $\begin{array}{l}\text { II }[9,13] \\
17.5(18.4)\end{array}$ & $\begin{array}{l}15[12,20] \\
20.0(19.5)\end{array}$ & $3.1 e-13$ & 2 & 0.006 & $S$ \\
\hline
\end{tabular}

Notes: Data are reported as median [95\% lower, upper confidence limits] and mean (standard deviation). Percentages describing smoking status and seropositivity refer only to patients' status at inclusion. P-values were calculated according to the chi-square test of independence for smoking status and proportion of seropositive patients, and the Kruskal-Wallis test for the remaining items. ${ }^{\mathrm{A}} \mathrm{P}$-values are ranked, within each time point individually (inclusion, I year and 8 years). ${ }^{\mathrm{B}}$ The Bonferroni-Holm adjustment - for multiple testing- was calculated for each time point individually $\alpha=0.05, n=$ number of tests, rank= rank of the exact $p$-values. Bonferroni-Holm adjusted level not shown for tests where the original $p$-value is not significant.

Abbreviations: ESR, erythrocyte sedimentation rate; GH-VAS, global health on a visual analogue scale; VAS, visual analogue scale; HAQ, Health Assessment Questionnaire, SHS, Sharp van der Heijde score; S, significant; NS, not significant.

significantly across age groups, but men $<40$ years had a significantly lower disease activity over time compared with men from other age groups (Figure 2B), due to lower ESR and GH-VAS (Tables 2 and 3). The DAS28 components are shown in Tables 2 and 3; ESR increased significantly with age at inclusion and after 1 and 8 years, for both sexes. Significant differences in GH-VAS between the age groups were detected only in men at 1 year. The number of swollen joints increased significantly with age at inclusion and after 1 year in women, but not in men. There was no significant difference in tender joints at inclusion, either in men or women. The number of tender joints increased very slightly, but significantly, with age in women at 1 and 8 years.

\section{Pain and Disability}

In both sexes and in all age groups, pain-VAS and HAQ decreased during the first year. After 1 year, pain-VAS was 
Table 3 Clinical Measures for Men at Inclusion, I Year and 8 Years

\begin{tabular}{|c|c|c|c|c|c|c|c|c|}
\hline Age Groups Total $n=921$ & $\begin{array}{l}<40 \text { Years } \\
n=78\end{array}$ & $\begin{array}{l}\text { 40-54 } \\
\text { Years } \\
n=198\end{array}$ & $\begin{array}{l}55-69 \\
\text { Years } \\
n=355\end{array}$ & $\begin{array}{l}\geq 70 \text { Years } \\
n=290\end{array}$ & p-value & $\begin{array}{l}\text { Rank of } \\
\text { p-value }\end{array}$ & $\begin{array}{l}\text { Bonferroni- } \\
\text { Holm } \\
\text { Adjusted } \\
\text { Significance } \\
\text { Level, } \alpha(a)^{B}\end{array}$ & $\begin{array}{l}\text { Significant } \\
\text { According } \\
\text { to } \\
\text { Bonferroni- } \\
\text { Holm } \\
p<\alpha(a)\end{array}$ \\
\hline $\begin{array}{l}\text { Symptom duration before } \\
\text { inclusion (mo) }\end{array}$ & $\begin{array}{l}6[5,7] \\
7.6(6.5)\end{array}$ & $\begin{array}{l}6[5,7] \\
8.3(8.4)\end{array}$ & $\begin{array}{l}6[5,6] \\
8.8(16.9)\end{array}$ & $\begin{array}{l}5[5,6] \\
6.9(11.3)\end{array}$ & 0.02 & 6 & 0.006 & NS \\
\hline $\begin{array}{c}\text { Smoking status (\%) } \\
\text { Current smoker } \\
\text { Previous smoker } \\
\text { Never smoker }\end{array}$ & $\begin{array}{l}19[10,27] \\
24[14,33] \\
57[47,69]\end{array}$ & $\begin{array}{l}38[31,44] \\
36[30,44] \\
26[19,31]\end{array}$ & $\begin{array}{l}45[39,49] \\
32[28,38] \\
23[19,28]\end{array}$ & $\begin{array}{l}50[43,55] \\
16[12,21] \\
34[29,40]\end{array}$ & $2.2 \mathrm{e}-\mathrm{II}$ & 1 & 0.004 & $S$ \\
\hline Seropositive (\%) & $72[6 I, 8 I]$ & $78[72,84]$ & $70[65,75]$ & $5 \mid[45,57]$ & $1.3 e-07$ & 4 & 0.005 & $S$ \\
\hline $\begin{array}{l}\text { ESR }(\mathrm{mm} / \mathrm{h}) \\
\text { Inclusion } \\
\text { I year } \\
8 \text { years }\end{array}$ & $\begin{array}{l}17[12,21] \\
23.8(21.1) \\
6[4,7] \\
10.4(11.6) \\
6[4,8] \\
10.0(12.6)\end{array}$ & $\begin{array}{l}24[22,26] \\
31.6(24.9) \\
10[9,11] \\
13.5(13.2) \\
12[10,14] \\
14.8(13.4)\end{array}$ & $\begin{array}{l}32[28,35] \\
37.4(25.3) \\
11[10,12] \\
17.6(17.4) \\
13[12,14] \\
19.4(18.9)\end{array}$ & $\begin{array}{l}35[32,39] \\
39.5(24.3) \\
14[12,16] \\
16[13,20]\end{array}$ & $\begin{array}{l}8.5 \mathrm{e}-09 \\
3.5 \mathrm{e}-08 \\
1.9 \mathrm{e}-08\end{array}$ & I & $\begin{array}{l}0.004 \\
0.006 \\
0.006\end{array}$ & S \\
\hline $\begin{array}{l}\text { GH-VAS }(0-100) \\
\text { Inclusion } \\
\text { I year } \\
8 \text { years }\end{array}$ & $\begin{array}{l}40[28,49] \\
39.2(26.6) \\
12[5,19] \\
18.1(20.1) \\
16[11,22] \\
21.5(22.6)\end{array}$ & $\begin{array}{l}47[44,50] \\
42.9(24.8) \\
24[19,27] \\
28.3(22.3) \\
18[14,22] \\
25.2(24.2)\end{array}$ & $\begin{array}{l}42[39,45] \\
41.5(24.9) \\
17[14,22] \\
23.6(22.7) \\
19[14,23] \\
25.8(24.7)\end{array}$ & $\begin{array}{l}41[36,46] \\
39.6(25.6) \\
14[8,18] \\
20.9(21.3) \\
15[8,20] \\
23.9(24.4)\end{array}$ & $\begin{array}{l}0.43 \\
3.0 \mathrm{e}-04 \\
0.54\end{array}$ & 4 & $\begin{array}{l}- \\
0.008\end{array}$ & $\begin{array}{l}\text { NS } \\
\text { S } \\
\text { NS }\end{array}$ \\
\hline $\begin{array}{l}\text { Tender joints (0-28) } \\
\text { Inclusion } \\
\text { I year } \\
8 \text { years }\end{array}$ & $\begin{array}{l}8[6,10] \\
8.5(6.1) \\
1[0,2] \\
2.6(3.6) \\
0[0,0] \\
1.4(3.0)\end{array}$ & $\begin{array}{l}7[6,8] \\
7.7(5.0) \\
I[1,2] \\
3.3(5.0) \\
0[0,0] \\
1.9(3.6)\end{array}$ & $\begin{array}{l}6[5,7] \\
7.5(6.4) \\
I[0, \mathrm{I}] \\
2.8(4.6) \\
0[0,0] \\
I .5(3.0)\end{array}$ & $\begin{array}{l}6[5,7] \\
7.6(6.6) \\
0[0,1] \\
2.1(3.8) \\
0[0,0] \\
\text { I.I }(2.9)\end{array}$ & $\begin{array}{l}0.22 \\
0.02 \\
0.20\end{array}$ & 7 & $\begin{array}{l}- \\
0.0167\end{array}$ & $\begin{array}{l}\text { NS } \\
\text { NS } \\
\text { NS }\end{array}$ \\
\hline $\begin{array}{l}\text { Swollen joints (0-28) } \\
\text { Inclusion } \\
\text { I year } \\
8 \text { years }\end{array}$ & $\begin{array}{l}\text { II }[10,12] \\
I 0.4(6.0) \\
I[0,2] \\
3.3(4.3) \\
0[0,0] \\
I .7(4.1)\end{array}$ & $\begin{array}{l}9[8,10] \\
9.8(5.3) \\
2[1,3] \\
3.5(4.2) \\
1[0,1] \\
2.3(3.5)\end{array}$ & $\begin{array}{l}10[9,1 \mathrm{I}] \\
11.1(6.1) \\
2[1,2] \\
3.3(4.7) \\
0[0,0] \\
2.0(3.5)\end{array}$ & $\begin{array}{l}\text { II }[10,12] \\
\text { II.6 (5.9) } \\
\text { I }[0,2] \\
2.7(3.8) \\
0[0,0] \\
I .3(2.2)\end{array}$ & $\begin{array}{l}0.03 \\
0.04\end{array}$ & $\begin{array}{l}7 \\
8\end{array}$ & $\begin{array}{l}0.007 \\
0.025 \\
0.007\end{array}$ & $\begin{array}{l}\text { NS } \\
\text { NS } \\
\text { NS }\end{array}$ \\
\hline $\begin{array}{c}\text { Pain }(0-100) \\
\text { Inclusion }\end{array}$ & $\begin{array}{l}42[35,51] \\
43.5(22.4)\end{array}$ & $\begin{array}{l}45[38,49] \\
44.6(23.8)\end{array}$ & $\begin{array}{l}43[38,47] \\
43.8(25.1)\end{array}$ & $\begin{array}{l}40[36,45] \\
40.8(24.5)\end{array}$ & 0.28 & II & - & NS \\
\hline $\begin{array}{l}\text { I year } \\
8 \text { years }\end{array}$ & $\begin{array}{l}12[6,19] \\
17.7(21.1) \\
14[5,19] \\
23.3(25.7)\end{array}$ & $\begin{array}{l}23[19,28] \\
27.6(22.5) \\
20[15,26] \\
27.5(24.8)\end{array}$ & $\begin{array}{l}16[14,20] \\
24.4(23.8) \\
19[14,23] \\
26.4(25.8)\end{array}$ & $\begin{array}{l}11[8,15] \\
19.3(20.8) \\
15[10,23] \\
23.8(24.3)\end{array}$ & 0.28 & 3 & 0.007 & $\begin{array}{l}\text { S } \\
\text { NS }\end{array}$ \\
\hline
\end{tabular}


Table 3 (Continued).

\begin{tabular}{|c|c|c|c|c|c|c|c|c|}
\hline Age Groups Total $n=921$ & $\begin{array}{l}<40 \text { Years } \\
n=78\end{array}$ & $\begin{array}{l}\text { 40-54 } \\
\text { Years } \\
n=198\end{array}$ & $\begin{array}{l}55-69 \\
\text { Years } \\
n=355\end{array}$ & $\begin{array}{l}\geq 70 \text { Years } \\
n=290\end{array}$ & p-value & $\begin{array}{l}\text { Rank of } \\
\text { p-value }\end{array}$ & $\begin{array}{l}\text { Bonferroni- } \\
\text { Holm } \\
\text { Adjusted } \\
\text { Significance } \\
\text { Level, } \alpha(a)^{B}\end{array}$ & $\begin{array}{l}\text { Significant } \\
\text { According } \\
\text { to } \\
\text { Bonferroni- } \\
\text { Holm } \\
p<\alpha(a)\end{array}$ \\
\hline \multicolumn{9}{|l|}{ HAQ (0-3) } \\
\hline Inclusion & $\begin{array}{l}0.8[0.5, \\
0.9] \\
0.8(0.5)\end{array}$ & $\begin{array}{l}0.8[0.6, \\
0.9] \\
0.8(0.5)\end{array}$ & $\begin{array}{l}0.9[0.8, \\
1.0] \\
0.9(0.6)\end{array}$ & $\begin{array}{l}0.8[0.7, \\
0.9] \\
0.9(0.7)\end{array}$ & 0.57 & 13 & - & NS \\
\hline I year & $\begin{array}{l}0.1[0.0, \\
0.1] \\
0.3(0.4)\end{array}$ & $\begin{array}{l}0.4[0.3, \\
0.5] \\
0.5(0.5)\end{array}$ & $\begin{array}{l}0.3[0.1, \\
0.4] \\
0.4(0.5)\end{array}$ & $\begin{array}{l}0.4[0.3, \\
0.5] \\
0.5(0.6)\end{array}$ & 0.01 & 6 & 0.013 & $S$ \\
\hline 8 years & $\begin{array}{l}0.0[0.0, \\
0.0] \\
0.3(0.4)\end{array}$ & $\begin{array}{l}0.4[0.3 \\
0.5] \\
0.5(0.5)\end{array}$ & $\begin{array}{l}0.3 \\
{[0.1,0.4]} \\
0.5(0.6)\end{array}$ & $\begin{array}{l}0.5[0.4, \\
0.8] \\
0.7(0.7)\end{array}$ & $2.2 \mathrm{e}-05$ & 2 & 0.006 & $S$ \\
\hline \multicolumn{9}{|l|}{ Joint destruction (SHS) } \\
\hline \multicolumn{9}{|l|}{ Total score $(0-448)$} \\
\hline Inclusion & $\begin{array}{l}0[0,0] \\
4.0(11.7)\end{array}$ & $\begin{array}{l}0[0,1] \\
3.4(8.6)\end{array}$ & $\begin{array}{l}2[1,3] \\
5.1(8.9)\end{array}$ & $\begin{array}{l}3[2,4] \\
6.7(9.6)\end{array}$ & & 5 & & S \\
\hline I year & $\begin{array}{l}2[0,3] \\
5.4(12.7)\end{array}$ & $\begin{array}{l}2[1,3] \\
6.5(10.6)\end{array}$ & $\begin{array}{l}4[3,5] \\
8.4(11.6)\end{array}$ & $\begin{array}{l}5[3,7] \\
9.1(11.8)\end{array}$ & 0.002 & 5 & 0.01 & S \\
\hline 8 years & $\begin{array}{l}12[3,16] \\
15.0(19.1)\end{array}$ & $\begin{array}{l}\text { II }[7, \text { I4] } \\
\text { I5.7 (I7.4) }\end{array}$ & $\begin{array}{l}13[10,16] \\
20.8(23.6)\end{array}$ & $\begin{array}{l}13[8,19] \\
18.5(19.2)\end{array}$ & 0.29 & 7 & - & NS \\
\hline \multicolumn{9}{|l|}{ Erosion score (0-280) } \\
\hline Inclusion & $\begin{array}{l}0[0,0] \\
\text { I.6 (4.5) }\end{array}$ & $\begin{array}{l}0[0,0] \\
\text { I.5 (4.I) }\end{array}$ & $\begin{array}{l}0[0,0] \\
2.1(4.4)\end{array}$ & $\begin{array}{l}0[0,0] \\
I .8(3.5)\end{array}$ & 0.24 & 10 & - & NS \\
\hline I year & $\begin{array}{l}0[0,1] \\
2.6(5.3)\end{array}$ & $\begin{array}{l}0[0,1] \\
2.8(5.2)\end{array}$ & $\begin{array}{l}\text { I }[0,2] \\
3.3(5.6)\end{array}$ & $\begin{array}{l}0[0,1] \\
2.9(5.2)\end{array}$ & 0.52 & 9 & - & NS \\
\hline 8 years & $\begin{array}{l}2[0,3] \\
4.7(8.6)\end{array}$ & $\begin{array}{l}2[1,3] \\
4.9(7.6)\end{array}$ & $\begin{array}{l}3[2,5] \\
6.9(9.9)\end{array}$ & $\begin{array}{l}\text { I }[0,2] \\
4.5(6.5)\end{array}$ & 0.31 & 8 & - & NS \\
\hline \multicolumn{9}{|l|}{ Joint space narrowing (0-168) } \\
\hline Inclusion & $\begin{array}{l}0[0,0] \\
2.3(8.1)\end{array}$ & $\begin{array}{l}0[0,0] \\
1.9(4.9)\end{array}$ & $\begin{array}{l}0[0, I] \\
3.1(5.5)\end{array}$ & $\begin{array}{l}2[0,3] \\
4.9(7.5)\end{array}$ & $5.0 e-08$ & 3 & 0.005 & $S$ \\
\hline I year & $\begin{array}{l}0[0,1] \\
2.8(8.3)\end{array}$ & $\begin{array}{l}\text { I }[0,2] \\
3.7(6.4)\end{array}$ & $\begin{array}{l}2[1,3] \\
5.0(7.5)\end{array}$ & $\begin{array}{l}4[3,6] \\
6.5(8.6)\end{array}$ & $3.0 \mathrm{e}-06$ & 2 & 0.006 & $S$ \\
\hline 8 years & $\begin{array}{l}7[2,12] \\
10.8(13.7)\end{array}$ & $\begin{array}{l}7[5,10] \\
10.8(12.5)\end{array}$ & $\begin{array}{l}9[7,12] \\
13.9(15.6)\end{array}$ & $\begin{array}{l}10[7,14] \\
14.2(14.7)\end{array}$ & 0.15 & 4 & - & NS \\
\hline
\end{tabular}

Notes: Data are reported as median [95\% lower, upper confidence limits] and mean (standard deviation). Percentages describing smoking status and seropositivity refer only to patients' status at inclusion. P-values were calculated according to the chi-square test of independence for smoking status and proportion of seropositive patients, and the Kruskal-Wallis test for the remaining items. ${ }^{A} \mathrm{p}$-values are ranked, within each time point individually (inclusion, I year and 8 years). ${ }^{\mathrm{B}}$ The Bonferroni-Holm adjustment - for multiple testing- was calculated for each time point individually. $\alpha=0.05, n=$ number of tests, rank= rank of the exact $p$-values Bonferroni-Holm adjusted level not shown for tests where the original p-value is not significant.

Abbreviations: ESR, erythrocyte sedimentation rate, GH-VAS, global health on a visual analogue scale, VAS, visual analogue scale, HAQ, Health Assessment Questionnaire, SHS, Sharp van der Heijde score; S, significant; NS, not significant.

relatively constant in both women and men within the age groups (Table 2 and Table 3). A significant difference between age groups was only seen in men at 1 year; men
$<40$ years and men $\geq 70$ years had the lowest scores and men 40-54 years had the highest. Men $<40$ years had significantly lower HAQ after one year compared with all 
Table 4 Clinical Outcomes, Given as Median Values, Compared Across Age Groups and Between Sexes

\begin{tabular}{|c|c|c|c|}
\hline Time Point, Age-Group & Women & Men & $\begin{array}{l}\text { P-value } \\
\text { Comparison } \\
\text { Between } \\
\text { Sexes }^{\mathbf{A}}\end{array}$ \\
\hline \multicolumn{4}{|l|}{ DAS28 } \\
\hline Inclusion, $<40$ yrs & 5.2 & 4.9 & NS \\
\hline Inclusion, $40-54$ yrs & 5.1 & 5.2 & NS \\
\hline Inclusion, 55-69 yrs & 5.5 & 5.2 & $<0.01$ \\
\hline Inclusion, $\geq 70$ yrs & 5.5 & 5.3 & $<0.001$ \\
\hline P-value within sex for age-groups ${ }^{A}$ & $<0.001$ & $0.3, \mathrm{NS}$ & \\
\hline I year, $<40$ yrs & 3.3 & 2.4 & $<0.001$ \\
\hline I year, 40-54 yrs & 3.4 & 3.1 & 0.03 \\
\hline I year, 55-69 yrs & 3.6 & 2.8 & $<0.001$ \\
\hline I year, $>70$ yrs & 3.4 & 2.8 & $<0.001$ \\
\hline P-value within sex for age-groups ${ }^{A}$ & 0.05 & 0.01 & \\
\hline 8 years, $<40$ yrs & 2.8 & 1.9 & $<0.001$ \\
\hline 8 years, $40-54$ yrs & 3 & 2.6 & $<0.01$ \\
\hline 8 years, $55-69$ yrs & 2.9 & 2.4 & $<0.001$ \\
\hline 8 years, $>70$ yrs & 3.1 & 2.5 & $<0.001$ \\
\hline P-value within sex for age-groups ${ }^{A}$ & $0.4, \mathrm{NS}$ & $<0.01$ & \\
\hline \multicolumn{4}{|l|}{ HAQ (0-3) } \\
\hline Inclusion, $<40$ yrs & 0.9 & 0.8 & $<0.01$ \\
\hline Inclusion, $40-54$ yrs & 1 & 0.8 & $<0.001$ \\
\hline Inclusion, 55-69 yrs & $\mathrm{I}$ & 0.9 & $<0.001$ \\
\hline Inclusion, $\geq 70$ yrs & 1.3 & 0.8 & $<0.001$ \\
\hline I year, $<40$ yrs & 0.4 & 0.1 & $<0.001$ \\
\hline I year, 40-54 yrs & 0.6 & 0.4 & $<0.001$ \\
\hline I year, 55-69 yrs & 0.6 & 0.3 & $<0.001$ \\
\hline I year, $>70$ yrs & 0.8 & 0.4 & $<0.001$ \\
\hline 8 years, $<40$ yrs & 0.4 & 0 & $<0.001$ \\
\hline 8 years, $40-54$ yrs & 0.6 & 0.4 & $<0.001$ \\
\hline 8 years, 55-69 yrs & 0.6 & 0.3 & $<0.001$ \\
\hline 8 years, $>70$ yrs & 0.9 & 0.5 & $<0.001$ \\
\hline \multicolumn{4}{|l|}{ Pain-VAS $(0-100)$} \\
\hline Inclusion, <40 yrs & 49 & 42 & 0.08 \\
\hline Inclusion, 40-54 yrs & 48 & 45 & 0.13 \\
\hline Inclusion, 55-69 yrs & 49 & 43 & $<0.01$ \\
\hline Inclusion, $\geq 70 \mathrm{yrs}$ & 47 & 40 & $<0.01$ \\
\hline I year, $<40$ yrs & 27 & 12 & $<0.001$ \\
\hline I year, 40-54 yrs & 28 & 23 & 0.08 \\
\hline I year, 55-69 yrs & 24 & 16 & $<0.001$ \\
\hline I year, >70 yrs & 25.5 & 11 & $<0.001$ \\
\hline 8 years, $<40$ yrs & 25 & 14 & 0.01 \\
\hline 8 years, $40-54$ yrs & 29 & 20 & $<0.01$ \\
\hline 8 years, 55-69 yrs & 25 & 19 & 0.01 \\
\hline 8 years, $>70$ yrs & 33 & 15 & $<0.001$ \\
\hline
\end{tabular}

(Continued)
Table 4 (Continued).

\begin{tabular}{|l|l|l|l|}
\hline Time Point, Age-Group & Women & Men & $\begin{array}{l}\text { P-value } \\
\text { Comparison } \\
\text { Between } \\
\text { Sexes }\end{array}$ \\
\hline SHS (0-448) & & & \\
Inclusion, <40 yrs & 0 & 0 & 0.13 \\
Inclusion, 40-54 yrs & 0 & 0 & 0.75 \\
Inclusion, 55-69 yrs & 2 & 2 & 0.64 \\
Inclusion, $\geq 70$ yrs & 4 & 3 & 0.06 \\
I year, <40 yrs & 0 & 2 & 0.59 \\
I year, 40-54 yrs & 2 & 2 & 0.68 \\
I year, 55-69 yrs & 5 & 4 & 0.15 \\
I year, >70 yrs & 8 & 5 & $<0.01$ \\
8 years, <40 yrs & 5 & 12 & 0.29 \\
8 years, 40-54 yrs & 11 & 11 & 0.53 \\
8 years, 55-69 yrs & 15 & 13 & 0.13 \\
8 years, >70 yrs & 19 & 13 & 0.05 \\
\hline
\end{tabular}

Note: ${ }^{A} P$-values are given according to the Mann-Whitney test.

Abbreviations: DAS28, 28-joint disease activity score, HAQ, Health Assessment Questionnaire, VAS, visual analogue scale, SHS, Sharp van der Heijde Score for joint destruction; NS, not significant.

other groups. HAQ was higher among women compared with men for all age groups and at all time points $(p<0.001$, Table 4). For both sexes, the most favorable HAQ was seen for those $<40$ years $(\mathrm{p}<0.001)$.

\section{Joint Destruction}

At one year after inclusion, 2613 (92\%) patients were assessed and of those $71 \%$ had radiographic assessment of hands and feet. At the 8-year follow-up, 2213 patients (78\%) were assessed and $73 \%$ of these had radiographic assessments.

The SHS differed significantly between age groups for women at all time points (Table 2) and at inclusion and 1 year for men (Table 3). Joint destruction increased steadily over time, and women $\geq 70$ years consistently had the highest SHS at all time points compared with all other groups. Men $<40$ years had less joint destruction at inclusion and at 1 year compared with all other groups (Table 3). There were no differences in SHS between men and women under the age of 70 years, whereas older 


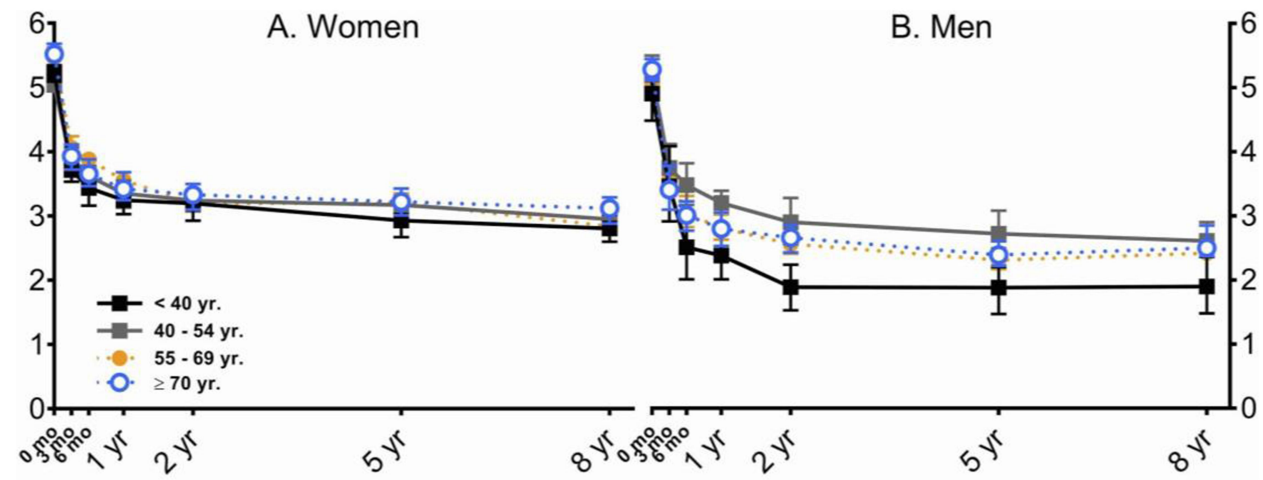

Figure 2 Median disease activity score over the course of the study. Disease activity in (A) women and (B) men over 8 years from diagnosis, measured using the composite disease activity score (DAS28) at all assessment time points for subjects divided by age group: black cube < 40 years; grey cube $40-54$ years; closed circle $55-69$ years; open circle $>70$ years. Symbols show median values, error bars represent $95 \%$ confidence intervals.

women had marginally higher SHS than older men (Table 4).

\section{Medical Treatment}

Figure 3 shows the percentage of patients receiving methotrexate only, sulfasalazine only or bDMARD alone or in combination (Figure $3 \mathrm{~A}-\mathrm{F}$ ) and/or GC (Figure $3 \mathrm{G}-\mathrm{H}$ ). Methotrexate was the most common csDMARD, and bDMARDs were the least common treatment at inclusion. The usage of bDMARDs increased over time and younger patients were more likely than older patients to receive bDMARDs. Treatment with GC, given alone or together with a DMARD, was similar across all age groups and between sexes, except that they were less often prescribed for men $<40$ years. Less than $20 \%$ of the patients received GC in monotherapy, however those patients were preferentially women and men $\geq 70$ years (Supplementary Figure 1$)$. At 8 years, men $\geq 70$ years had, compared with all other groups, the highest proportion of no treatment, ie neither DMARD nor GC.

\section{Discussion}

This study investigated the influence of sex and age of onset on the disease course and treatment of more than 2000 early RA patients during 8 years. The striking result that emerges is the great consistency of disease courses measured (Figure 2) in view of the variety of management strategies covered (Figure 3, Supplementary Figure 1). All patients showed significant improvement in most clinical measures immediately on commencement of treatment and throughout the first year, after which disease activity (DAS28) remained fairly constant. Nonetheless, we find that our choice of 4 age bands, formulated specifically to match the maturation of hormone profiles in patients of both sexes, not only provides a useful breakdown of the stages of disease ensuing for patients of different age at onset, but also reveals sex differences that may otherwise be masked.

The notable example of such a sex difference is in the group of men $<40$ years old at onset: from 6 months and onwards, they showed more improvement in DAS28 than all other groups, both male and female, despite there being no significant difference at inclusion. Nevertheless, at 8 years, there was no difference in joint destruction compared to older men. However, this is the age group that was least represented in our study $(n=78$, representing $9 \%$ of the male participants and only $3 \%$ of the entire BARFOT cohort) and these results should be interpreted with care, as these findings might be confounded by the low sample size. At inclusion, women older than 55 years had a significantly elevated median DAS28 compared with age-matched men. During the eight years of follow-up, there was almost no difference in DAS28 between the age groups in women, whereas men younger than 40 years consistently had a lower DAS28 compared with the other age-groups. Tender and swollen joint count decreased strongly and consistently throughout the followup and were between 0 and 1 for all groups at 8 years. As previously reported, ${ }^{4}$ the remaining components of DAS28, ESR and GH-VAS, had a greater impact on DAS28 and might not reflect just joint inflammation. It is however noteworthy that ESR in both men and women falls dramatically on commencement of treatment, in all age groups. Moreover, our results are in line with the knowledge that ESR increases with age, particularly in women, ${ }^{21}$ which could mean that the higher DAS28 in 

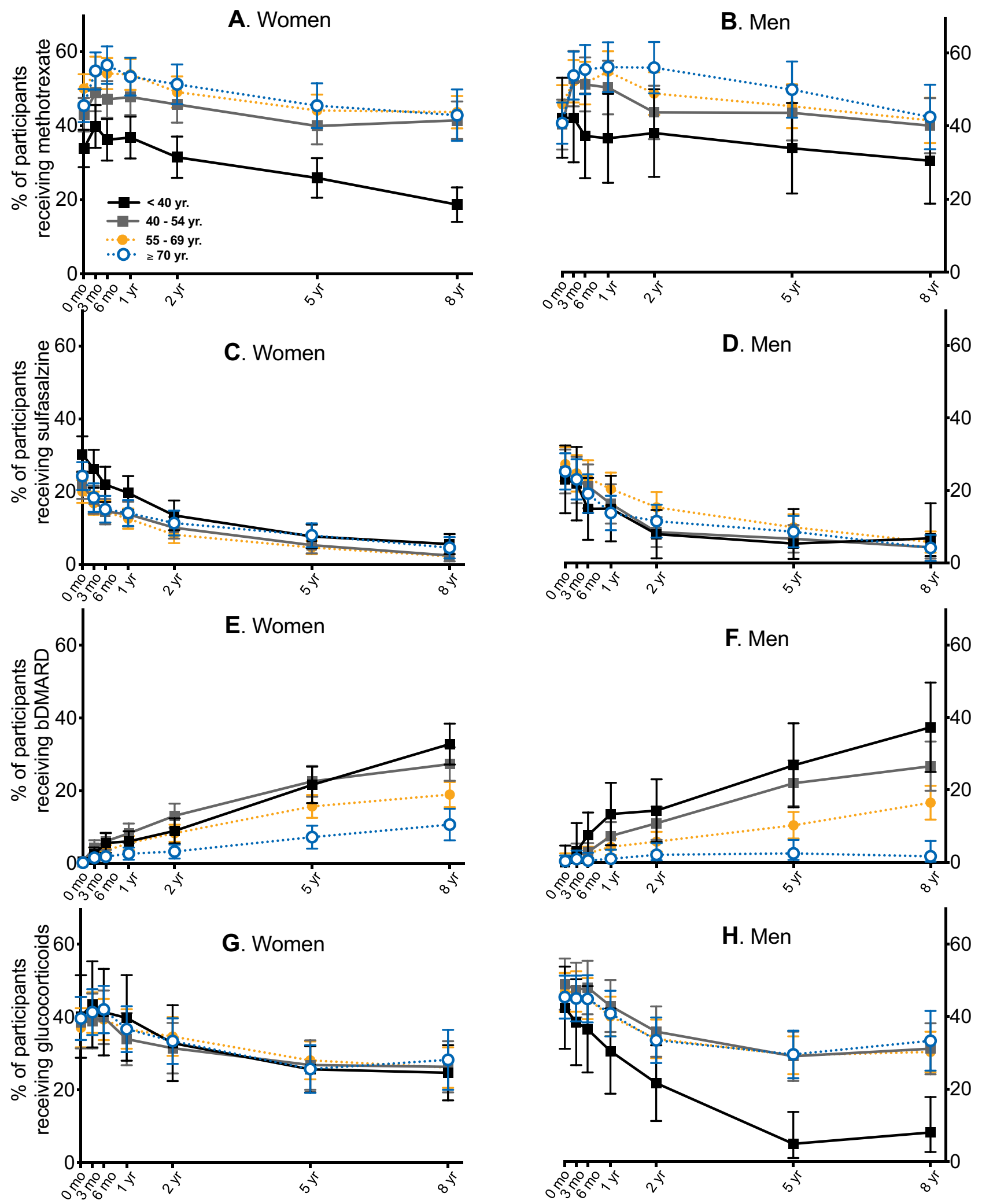

Figure 3 Distribution of drug treatments amongst the study population. Percentages of subjects receiving DMARD and/or glucocorticoid treatment over the course of the study are given for methotrexate (A and $\mathbf{B}$ ), sulfasalazine (C and $\mathbf{D})$, biological DMARDs (E and $\mathbf{F}$ ) and glucocorticoids (both alone and in combination with DMARDs) (G and H). Panels (A, C, E, G) show data from women, panels (B, D, F, H) are from men; subjects are divided by age group: black cube < 40 years; grey cube $40-54$ years; closed circle 55-69 years; open circle $>70$ years. Symbols show median values, error bars represent $95 \%$ confidence intervals. 
older women was probably attributed to the measures of disease activity rather than to the disease activity per se. Thus, differences in age and sex should be taken into account when interpreting DAS28.

Women generally had higher HAQ compared with men as has been previously found. ${ }^{22,23}$ Camacho et al found that a sex-independent higher rate of increased disability (HAQ) associated with increased age of symptom onset. The same study showed that after 5 years the HAQ scores were higher than at baseline for patients 55 years and older. ${ }^{22}$ Arnold et al $^{13}$ found that older-onset patients ( $\geq 64$ years) start and end the first year after diagnosis worse in terms of DAS28 and HAQ compared with younger patients. In the current study, irrespective of age, HAQ decreased during the first year and was thereafter relatively stable Women $\geq 70$ years showed a slight increase after 1 year but the HAQ scores at 8 years were still below baseline level at inclusion.

In contrast to all other measures, radiographically assessed joint destruction increased steadily over time. In women, the SHS increased significantly with age group at all time points, mainly due to joint space narrowing. However, joint space narrowing is not specific for RA. Coexisting osteoarthritis has been shown to contribute to a higher joint space narrowing score at inclusion in patients over 55 years of age relative to their younger counterparts. ${ }^{24}$ In men, the increase with age was significant at inclusion and after one year but this difference was no longer evident at the 8 years timepoint. Previously, Ahlmén et $\mathrm{al}^{25}$ found no significant differences in SHS between women and men, whereas other studies found men to have a greater radiological destruction at 24 months compared with women. ${ }^{12}$ In the present study, we found no significant differences between men and women, except for the $\geq$ 70 years women who had higher SHS than men at all time points. Further, this group had consistently the highest SHS among women. There was no difference in the erosion score for men and only a marginal increase for women older than 55 years at one-year follow-up. This indicates that the SHS changes seen in women $\geq 70$ years were mainly due to increased joint space narrowing. Hence, by introducing age as a factor, the different trajectories of changes in joint architecture experienced by men and women are disclosed.

For the individual patient, treatment is chosen after careful considerations that include disease activity, age, estimated prognosis, desire to have children for those of reproductive age, and comorbidities. As expected, methotrexate was by far the most commonly used DMARD in the BARFOT cohort. We found that the proportion of younger patients, in particular women less than 40 years, who were treated with methotrexate was lower compared with all other age groups. This could be due to the fact that Methotrexate has teratogenic side effects and is not prescribed before conception or during pregnancy and lactation. ${ }^{26}$ bDMARDs were introduced during the collection of the BARFOT cohort, which means that only the patients included during 1999-2006 (about half of the patients) had access to treatment with bDMARDs. As seen in other studies, ${ }^{11-14}$ the prescription of bDMARDs was highest for younger patients and decreased with age at onset. GC in monotherapy was used in $<20 \%$ of the patients, however, the patients who were most likely to receive this treatment were women and men $\geq 70$ years. Patients younger than 40 years were the least likely to receive $\mathrm{GC}$ as the only treatment. Otherwise, no differences for GC as monotherapy or in combination with a DMARD were found. Our findings are in coherence with an epidemiological study on DMARD prescription, covering $87 \%$ of the German population, which found that treatment during the first year after onset of RA differed with respect to both sex and age; ${ }^{14}$ women were less likely to receive cDMARD, bDMARD and GC during the first year compared with men. The same study found that patients $\geq$ 65 years were less likely to receive DMARDs and more likely to receive only GC compared with patients $<35$ years even though these patients had the highest DAS28 levels. ${ }^{4}$ These findings were contradicted in a large multinational cohort of patients with manifest RA, where no differences in treatment strategies between the sexes were found even though women had a worse disease outcome. ${ }^{3}$ One possible explanation for $\mathrm{GC}$ as monotherapy is the occurrence of comorbidities, since RA patients with late onset have a heavier burden of comorbidities compared with younger ones. ${ }^{11}$ Notably, GC treatment has been reported to be more common in patients with late onset. ${ }^{11,12}$

Access to bDMARDs and targeted synthetic DMARDs have improved the disease outcome immensely and today the treatment aim is clinical remission, or at least low disease activity. ${ }^{7}$ The perfect treatment is an act of balance between too little and too much immunosuppression, and particularly among older RA patients there is often 
a tension between the likely benefit of treating the active disease and the risk of adverse events due to frailty or comorbidities. $^{27,28}$

Separating out those participants who were $>70$ years old at diagnosis confirmed previous reports that the frequency of RF was lower in the elderly, who more frequently had large joint involvement and disease distribution was more equal between the sexes. ${ }^{1,29}$ Thus, our study reinforces the question posed by other previous studies whether RA in elderly is the same disease as RA in younger persons. ${ }^{12,13}$

Our study has some limitations. The patients were collected to the BARFOT cohort 1992-2006 and treatment strategies as well as classification criteria have changed. Long-term follow-up studies of RA outcomes are necessary and these limitations are unavoidable. As shown earlier, the patients included in the 2000s (half of the patients) were more often treated with MTX and biologics than those included in 1990s. ${ }^{30}$ However, despite greater reduction in disease activity for the more actively treated patients, the gender difference persisted during 8 years, with men achieving lower DAS28 than women. Therefore we suggest the present findings also to be valid for more actively treated patients and more recent cohorts.

Comorbidities such as hypertension and diabetes may have influenced at least partially the responsiveness to glucocorticoids. All patients, irrespective of comorbidity, were included in the study. However, the information regarding comorbidities was not consistently recorded, which makes it impossible to estimate their impact on our results.

Of the participants present at inclusion, $22 \%$ were not available for the 8-year examination. The nonparticipating patients were on average 11 years older at inclusion and more likely to be men. They did not differ from the participants in regard to any of the disease activity indicators, with the exception of JSN at inclusion (median 2 vs 0 in the participants, $\mathrm{p}<0.001$ ) which is non-specific for RA and may indicate comorbidity for osteoarthritis and associate with higher age. Taken together, the loss of non-participants might have rendered our findings regarding age differences at 8 -years as slightly conservative, potentially more so for men. The BARFOT cohort encompasses more than 2800 patients. However, the division of the patients into eight subgroups resulted in small numbers of participants in some of the subgroups, especially for the younger men which is an important group for the purpose of this study. This shortcoming limited both the number of statistical analyses we were able to perform and the power of those we performed, and the results regarding younger men should be interpreted with caution. Nonetheless, we consider this approach to have provided us with useful insights.

The aim of this study was to investigate the influence of sex and age on disease development and treatment choices, but our understanding of the influence of age alone on disease development is blurred by the variety of treatment choices across age groups in the cohort.

\section{Conclusions}

This large cohort study of patients with early RA over the 8 years following their diagnosis reveals that the disease course for all ages and both sexes was similar, regardless of the details of treatments given, but useful insight was provided by differential analysis using specific function-related age bands and sex as factors.

Men $<40$ years of age had the most favorable functional outcome over the study period. The significantly lower DAS28 over time was manifest despite no obvious difference at diagnosis. This group (together with women $<40$ years) was also more likely to be treated with bDMARDs. Results for this group should be cautiously interpreted due to the low sample size.

Women $\geq 70$ years experienced the smallest improvement with respect to disability and joint destruction over the study period, and were, together with men in the same age group, more likely to receive treatment with only GC.

Whether the differences in outcome are due to the phenotype of the disease or its treatment is not clear. However, late age onset might be a risk factor for a worse disease outcome and appropriate treatment is important irrespective of age. Our results indicate that there is a need for specific treatment guidelines for patients older than 70 years.

\section{Acknowledgment}

Kristina Albertsson, for assessment of radiographs, and the BARFOT Study Group.

\section{Disclosure}

The authors report no conflicts of interest in this work. 


\section{References}

1. Eriksson JK, Neovius M, Ernestam S, Lindblad S, Simard JF, Askling J. Incidence of rheumatoid arthritis in Sweden: a nationwide population-based assessment of incidence, its determinants, and treatment penetration. Arthritis Care Res (Hoboken). 2013;65(6):870-878. doi:10.1002/acr.21900

2. Tengstrand B, Ahlmen M, Hafstrom I. The influence of sex on rheumatoid arthritis: a prospective study of onset and outcome after 2 years. J Rheumatol. 2004;31(2):214-222.

3. Sokka T, Toloza S, Cutolo M, et al. Women, men, and rheumatoid arthritis: analyses of disease activity, disease characteristics, and treatments in the QUEST-RA study. Arthritis Res Ther. 2009;11(1): R7. doi:10.1186/ar2591

4. van Onna M, Boonen A. The challenging interplay between rheumatoid arthritis, ageing and comorbidities. BMC Musculoskelet Disord. 2016;17(1):184. doi:10.1186/s12891-016-1038-3

5. Eriksson JK, Johansson K, Askling J, Neovius M. Costs for hospital care, drugs and lost work days in incident and prevalent rheumatoid arthritis: how large, and how are they distributed? Ann Rheum Dis. 2015;74(4):648-654. doi:10.1136/annrheumdis-2013-204080

6. Singh JA, Saag KG, Bridges SL Jr., et al. 2015 American college of rheumatology guideline for the treatment of rheumatoid arthritis. Arthritis Rheumatol. 2016;68(1):1-26. doi:10.1002/art.39480

7. Smolen JS, Landewe RBM, Bijlsma JWJ, et al. EULAR recommendations for the management of rheumatoid arthritis with synthetic and biological disease-modifying antirheumatic drugs: 2019 update. Ann Rheum Dis. 2020;79(6):685-699. doi:10.1136/annrheumdis-2019216655

8. van Hulst LT, Kievit W, van Bommel R, van Riel PL, Fraenkel L. Rheumatoid arthritis patients and rheumatologists approach the decision to escalate care differently: results of a maximum difference scaling experiment. Arthritis Care Res (Hoboken). 2011;63 (10):1407-1414. doi:10.1002/acr.20551

9. Ogasawara M, Tamura N, Onuma S, et al. Observational cross-sectional study revealing less aggressive treatment in Japanese elderly than nonelderly patients with rheumatoid arthritis. J Clin Rheumatol. 2010;16 (8):370-374. doi:10.1097/RHU.0b013e3181fe8b37

10. Tutuncu Z, Reed G, Kremer J, Kavanaugh A. Do patients with older-onset rheumatoid arthritis receive less aggressive treatment? Ann Rheum Dis. 2006;65(9):1226-1229. doi:10.1136/ard.2005.051144

11. Oishi S, Wendling D, Sibilia J, et al. Treatment of active rheumatoid arthritis: comparison of patients younger vs older than 75 years (CORPUS cohort). Hum Vaccin Immunother. 2018;14 (11):2612-2617. doi:10.1080/21645515.2018.1522470

12. Innala L, Berglin E, Moller B, et al. Age at onset determines severity and choice of treatment in early rheumatoid arthritis: a prospective study. Arthritis Res Ther. 2014;16(2):R94. doi:10.1186/ar4540

13. Arnold MB, Bykerk VP, Boire G, et al. Are there differences between young- and older-onset early inflammatory arthritis and do these impact outcomes? An analysis from the CATCH cohort. Rheumatology (Oxford). 2014;53(6):1075-1086. doi:10.1093/rheumatology/ket449

14. Steffen A, Holstiege J, Klimke K, Akmatov MK, Batzing J. Patterns of the initiation of disease-modifying antirheumatic drugs in incident rheumatoid arthritis: a German perspective based on nationwide ambulatory drug prescription data. Rheumatol Int. 2018;38 (11):2111-2120. doi:10.1007/s00296-018-4161-7

15. Hafstrom I, Ajeganova S, Andersson ML, et al. A Swedish register-based, long-term inception cohort study of patients with rheumatoid arthritis - results of clinical relevance. Open Access Rheumatol. 2019;11:207-217. doi:10.2147/OARRR.S218448
16. Arnett FC, Edworthy SM, Bloch DA, et al. The American Rheumatism Association 1987 revised criteria for the classification of rheumatoid arthritis. Arthritis Rheum. 1988;31(3):315-324. doi: 10.1002/art.1780310302

17. Prevoo ML, van 'T Hof MA, Kuper HH, van Leeuwen MA, van de Putte LB, van Riel PL. Modified disease activity scores that include twenty-eight-joint counts. Development and validation in a prospective longitudinal study of patients with rheumatoid arthritis. Arthritis Rheum. 1995;38(1):44-48. doi:10.1002/ art. 1780380107

18. Ekdahl C, Eberhardt K, Andersson SI, Svensson B. Assessing disability in patients with rheumatoid arthritis. Use of a Swedish version of the stanford health assessment questionnaire. Scand J Rheumatol. 1988;17(4):263-271. doi:10.3109/03009748809098795

19. van der Heijde D. How to read radiographs according to the Sharp/ van der Heijde method. J Rheumatol. 2000;27(1):261-263.

20. Armstrong RA. When to use the Bonferroni correction. Ophthalmic Physiol Opt. 2014;34(5):502-508. doi:10.1111/opo.12131

21. Miller A, Green M, Robinson D. Simple rule for calculating normal erythrocyte sedimentation rate. Br Med J (Clin Res Ed). 1983;286 (6361):266. doi:10.1136/bmj.286.6361.266

22. Camacho EM, Verstappen SM, Lunt M, Bunn DK, Symmons DP. Influence of age and sex on functional outcome over time in a cohort of patients with recent-onset inflammatory polyarthritis: results from the Norfolk Arthritis Register. Arthritis Care Res (Hoboken). 2011;63 (12):1745-1752. doi:10.1002/acr.20609

23. Andersson ML, Forslind K, Hafstrom I. Comparing five year out-come in two cohorts of patients with early rheumatoid arthritis - A BARFOT Study. Open Rheumatol J. 2015;9(1):8-15. doi:10.2174/1874312901409010008

24. Khanna D, Ranganath VK, Fitzgerald J, et al. Increased radiographic damage scores at the onset of seropositive rheumatoid arthritis in older patients are associated with osteoarthritis of the hands, but not with more rapid progression of damage. Arthritis Rheum. 2005;52 (8):2284-2292. doi:10.1002/art.21221

25. Ahlmen M, Svensson B, Albertsson K, Forslind K, Hafstrom I. Influence of gender on assessments of disease activity and function in early rheumatoid arthritis in relation to radiographic joint damage. Ann Rheum Dis. 2010;69(1):230-233. doi:10.1136/ard.2008.102244

26. Favalli EG, Biggioggero M, Crotti C, Becciolini A, Raimondo MG, Meroni PL. Sex and management of rheumatoid arthritis. Clin Rev Allergy Immunol. 2019;56(3):333-345. doi:10.1007/s12016-018-8672-5

27. Serhal L, Lwin MN, Holroyd C, Edwards CJ. Rheumatoid arthritis in the elderly: characteristics and treatment considerations. Autoimmun Rev. 2020;19(6):102528. doi:10.1016/j.autrev.2020.102528

28. Frisell T, Baecklund E, Bengtsson K, et al. Patient characteristics influence the choice of biological drug in RA, and will make non-TNFi biologics appear more harmful than TNFi biologics. Ann Rheum Dis. 2018;77 (5):650-657. doi:10.1136/annrheumdis-2017-212395

29. Kobak S, Bes C. An autumn tale: geriatric rheumatoid arthritis. Ther Adv Musculoskelet Dis. 2018;10(1):3-11. doi:10.1177/ $1759720 X 17740075$

30. Andersson MLE, Forslind K, Hafstrom I. Patients with early rheumatoid arthritis in the 2000s have equal disability and pain despite less disease activity compared with the 1990s: data from the BARFOT Study over 8 years. J Rheumatol. 2017;44(6):723-731. doi:10.3899/jrheum. 161235 


\section{Publish your work in this journal}

Open Access Rheumatology Research and Reviews is an international, peer-reviewed, open access journal publishing original research, reports, editorials, reviews and commentaries on all aspects of clinical and experimental rheumatology in the clinic and laboratory including the following topics: Pathology, pathophysiology of rheumatological diseases; Investigation, treatment and management

of rheumatological diseases; Clinical trials and novel pharmacological approaches for the treatment of rheumatological disorders. The manuscript management system is completely online and includes a very quick and fair peer-review system, which is all easy to use. Visit http://www.dovepress.com/testimonials.php to read real quotes from published authors. 